\title{
Acteurs et agents, points de vue géographiques au sein des sciences sociales
}

\section{Veronica Noseda et Jean-Bernard Racine}

\section{(2) OpenEdition}

\section{Journals}

Édition électronique

URL : http://journals.openedition.org/ress/647

DOI : $10.4000 /$ ress. 647

ISSN : 1663-4446

Éditeur

Librairie Droz

\section{Édition imprimée}

Date de publication : 1 novembre 2001

Pagination : 65-79

ISBN : 2-600-00663-X

ISSN : 0048-8046

\section{Référence électronique}

Veronica Noseda et Jean-Bernard Racine, « Acteurs et agents, points de vue géographiques au sein des sciences sociales ", Revue européenne des sciences sociales [En ligne], XXXIX-121 | 2001, mis en ligne le 11 décembre 2009, consulté le 10 décembre 2020. URL : http://journals.openedition.org/ress/ 647 ; DOI : https://doi.org/10.4000/ress.647 


\section{ACTEURS ET AGENTS, POINTS DE VUE GÉOGRAPHIQUES AU SEIN DES SCIENCES SOCIALES}

\section{EN GUISE D’AVANT-PROPOS INTRODUCTIF}

Dans son texte de présentation, Jean-Pierre Gaudin brasse de façon succincte mais très efficace la problématique de l'acteur. S'interroger sur ce que c'est qu'un acteur revient à ouvrir un éventail de questions qui sont au cœur de toute problématique scientifique dans les sciences sociales, et notamment à mettre le doigt sur l'arbitraire de toute une série d'oppositions qui jouissent néanmoins encore d'une fortune incontestée: individu contre société, subjectivisme contre objectivisme, liberté contre contrainte, micro contre macro, etc. Oppositions dont on sait qu'elles ont jalonné toute l'histoire des sciences sociales: si les «Pères fondateurs» affirmaient le plus souvent le primat de la société sur le sujet, l'heure aujourd'hui est au «retour du sujet» et à l'individu tout-puissant du post-modernisme. Il reste que depuis les années 60, maints auteurs ont essayé de montrer l'artificialité de ces «polarités irréelles» (Elias, 1997). Jean-Pierre Gaudin cite les plus importants: outre Norbert Elias, qui a posé, avec l'élaboration du concept d'habitus et d'habitus collectif, le problème du rapport entre individu et société en termes de processus dialectique et de configurations relationnelles, il mentionne les contributions théoriques de Pierre Bourdieu, de Jean-Claude Passeron, de Claude Grignon et d'Antony Giddens, qui ont tous démontré de façon convaincante l'apport heuristique d'une approche relationnelle du social.

Dans ces conditions, on pourrait se demander pourquoi le débat reste malgré tout d'actualité. Ne faut-il pas chercher les facteurs explicatifs dans la «nature» de l'acte scientifique en sciences sociales, qui se fonde sur un effort d'objectivation de la réalité sociale, mais qui ne peut en tout cas jamais être neutre? En rappelant les mots de Danièle Loshak, on peut dire que: «Un concept ne peut être neutre, parce qu'il n'est pas de lecture neutre du réel, que cette lecture est toujours dans la double dépendance de la subjectivité de celui qui l'effectue et des représentations collectives dominantes dans une culture donnée; mais aussi parce que son utilisation suggère subrepticement une lecture du réel de préférence à une autre, impose une grille d'interprétation du réel qui rejaillit nécessairement en aval sur les pratiques sociales. En effet, les mots ont une influence sur l' idée que nous nous faisons des choses, puisque c' est par la médiation du langage, et jamais directement, que nous percevons le monde; cela est particulièrement vrai des concepts-courants ou élaborés-à l'aide desquels nous décrivons ou analysons la réalité pour lui donner un sens» (Loshak, 1986).

Or, ce constat d'impossibilité de neutralité absolue ne doit pas nous empêcher de continuer dans la pratique réflexive que chaque bon chercheur doit effecteur 
sur son propre travail, en «objectivant» notamment sa propre position dans l'espace social ${ }^{1}$. Au contraire. Mais il devrait peut-être nous faire comprendre qu'une attitude angélique par rapport au champ intellectuel qui est le nôtre ne sert en rien la science: les sciences sociales sont traversées, comme tout autre domaine du social, par des luttes qui ont comme enjeu principal le sens à attribuer aux actions sociales, et le rôle des acteurs sociaux, soient-ils individuels ou collectifs. Les «polarités» mentionnées plus haut ne se situent pas qu'au niveau scientifique, mais aussi au niveau idéologique. Comme l'écrivaient les auteurs du Métier de sociologue: «les oppositions épistémologiques ne prennent tout leur sens que lorsqu'on les rapporte au système de positions et d'oppositions qui s'établissent entre des institutions, des groupes ou des cliques différemment situés dans le champ intellectuel. L'ensemble des caractéristiques qui définissent chaque chercheur, à savoir son type de formation (...), son statut dans l' université ou par rapport à l' université, ses appartenances institutionnelles, ses affiliations d' intérêt et sa participation à des groupes de pression proprement intellectuels (...), concourt à déterminer ses chances d'occuper telle ou telle position, c'est-à-dire d'épouser telles ou telles oppositions, dans le champ épistémologique» (Bourdieu, Chamboredon, Passeron; 1968). Autant dire que les réflexions sur les acteurs peuvent avoir ou reposer sur des enjeux autres que scientifiques.

Stimulé par le thème proposé pour ces rencontres, le géographe qui s'interroge ici sur ses propres pratiques et celles de ses collègues, et plus précisément sur le statut qu'ils ont donné aux acteurs, agents ou autres actants, dans la mesure du moins où ils en ont explicité l'existence et les relations, s'est associé à son assistante, politiste à l'origine, mais préparant une thèse de doctorat en géographie sur les problèmes de la violence urbaine. Leur commune relecture du texte introductif de Jean-Pierre Gaudin les a conduit à se poser une série de problèmes actuels auxquels ils se sont l'un et l'autre découverts confrontés, et dont le repérage manifeste bien, d'ailleurs, l'actualité du problème que cette question pose à l'ensemble des sciences sociales. Ils en resteront ici à trois séries de questions principales, en partant du plus général pour se rapprocher de préoccupations plus immédiatement liées à leur travail. La question de la distinction entre agent et acteur: de quoi s'agit-il? Sert-elle à quelque chose? La question de l'usage qu'ils pouvaient en faire en géographie, la question de «l'acteur géographique », dont on dit si volontiers qu'il façonne le territoire en même temps qu'il est, partiellement, façonné par lui, la question enfin de la ville, à laquelle on impute tout aussi facilement un rôle d'acteur, comme c'est le cas, par exemple, quand on évoque, à propos de violences urbaines, les «banlieues françaises ». Peut-on considérer la ville comme un acteur récent, actif sur la scène publique? Question qu'un géographe ne peut pas feindre d'ignorer, vu les répercussions qu'une réponse peut avoir sur la conception des politiques publiques, notamment. L'esquisse de réponse proposée nous permettra de retrouver, in fine et peut-être autrement, la question de la place des

«[...] le réalisme réflexif fait appel à une sociologie des intellectuels et à une sociologie de la science qui, loin de trahir un ethnocentrisme complaisant, sont des moyens essentiels de décentrement pour la sociologie dans son ensemble: on ne peut pas exprimer d'assertion relativement à un objet que lorsqu'on s'est affranchi de présupposés en les objectivant, en les situant dans une histoire qui est celle d'institutions, de traditions intellectuelles nationales, etc.» (Pinto, 1998: 147). 
acteurs dans une pratique de la recherche et du discours géographiques. Au total un ensemble de réflexions dont on espère qu'elles pourraient contribuer à nous permettre de franchir une étape ultérieure dans l'analyse réflexive de nos propres disciplines et de renouer avec des problèmes «fondamentaux» des sciences sociales qu'une attitude bien trop arrogante pourrait considérer comme résolus une fois pour toutes.

\section{LA DISTINCTION ENTRE AGENT ET ACTEUR: DE QUOI S'AGIT-IL? SERT-ELLE À QUELQUE CHOSE?}

Acteur et agent. En fait l'un a engendré l'autre. Etymologiquement du moins. Il est peut-être amusant de relever ici avec Roger Brunet (1992) dans Les mots de la géographie, l'étymologie du mot acteur: celui qui agit, de l'indo-européen ago qui était aussi conduire, mener (le troupeau) et a donné aussi agent, agiter, axiome, essai, examen, sans parler, plus étonnant peut-être, de synagogue, démagogue et ambigu. Autant dire avec lui que «l'action est multiforme». Mais qui dit action dit acteurs. Le mot n'effrayait pas le géographe.

\section{Les ingrédients d'une définition et d'une distinction possibles}

Si l'on feuillette différents dictionnaires de sciences sociales, et consulte l'entrée «acteur social» (le plus souvent liée à «action sociale») on tombe le plus souvent sur des définitions qui réunissent les éléments suivants:

1. L'acteur est un individu considéré en tant que composante de «systèmes sociaux » et agissant en conformité des normes et aux attentes qui convergent sur lui en tant qu'occupant une position sociale déterminée.

2. En ce sens, l'expression désigne le sujet d'un rôle et renvoie à la métaphore récurrente du theatrum mundi, selon laquelle la vie est un théâtre dans lequel chacun joue son rôle par rapport à son âge, son sexe, à la culture d'appartenance (le terme «rôle» est en effet la contraction du terme latin rotolus, le rouleau sur lequel l'acteur dans l'antiquité lisait sa partie sur scène).

3. Chaque théorie sociale incorpore une image plus ou moins explicite de l'acteur social. Les théories de l'action sociale oscillent entre un pôle volontariste, qui voit dans l'individu l'organisateur des éléments du système d'action, et un pôle déterministe qui voit dans l'homme un exécuteur de rôles.

C'est bien sur ce dernier point que nous aimerions concentrer notre attention. Cette « oscillation entre un pôle plus volontariste et un pôle plus déterministe», est souvent «résumée» par l'utilisation de deux termes différents, celui d'acteur et celui d'agent. Reprenons la définition de Pierre Ansart, tiré du vocabulaire sociologique édité avec André Akoun: «Le vocabulaire sociologique tend à distinguer les deux concepts d' agent et d'acteur. Par agent, on désigne l' individu qui exécute une tâche, qui est essentiellement déterminé à agir par des conditions qui lui sont imposées. Par acteur, on désigne l'individu qui agit selon ses propres desseins, selon ses propres motivations. Le fait de jouer un rôle dans une organisation ou 
une bureaucratie n' exclut pas que l' individu puisse se comporter en acteur: dans la mesure où il exécute le rôle prescrit selon ses propres besoins en l'adaptant ou en modifiant les interactions dans lesquelles il se trouve impliqué, il se comporte, dans une certaine mesure, en acteur. À certaines conditions, ce terme peut-être appliqué à un collectif. Une entreprise, une institution qui coordonnent les comportements de leurs membres dans des actions communes et assurent une continuité à leurs initiatives constituent des acteurs de la vie économique ou institutionnelle» (Akoun et Ansart, 1999)

Deux éléments de cette définition paraissent particulièrement intéressants. Le premier est, justement, la distinction entre agent et acteur, dont on essaiera d'évaluer la portée un peu plus loin. Le deuxième concerne l'introduction, au sein du «conflit titanesque» qui semble opposer individu et société, d'un troisième acteur: l'«acteur collectif». Le distinguer de l'agent devient dès lors essentiel, encore que les choses ne soient pas toujours très claires malgré les efforts des uns et des autres. On soulignera ici les efforts du sociologue Jean Remy (1989) qui, dans un travail sur la problématisation du changement social, insistant sur le fait que pour comprendre la séquence à long termes induite par le mouvement social, s'impose de distinguer le niveau de l'agent de celui de l'acteur. Certes «l' agent est un individu ou un groupe concret, comme le seraient les militaires ou la bourgeoisie terriennes». On peut donc, comme tel, en faire l'histoire dans des situations et des générations successives. Il reste que «lorsqu' au sein d' une transaction sociale, surgit une volonté de promouvoir un sens nouveau, s'enclenche une dynamique complémentaire. Lorsque la promotion du sens aboutit à la formation de solidarités expressives, l'action tend à se dédoubler, le niveau de l'agent étant transfiguré par une référence à l'acteur». Et Jean Remy d'évoquer la situation limite, "prototype d'une mobilisation forte», "d' un agent concret qui s'exprime de façon monopolistique une position actorielle», «le cas au XIXe siècle pour les mouvements nationaux et les mouvements ouvriers». Sans éviter de noter pourtant que "certains font l' hypothèse que la complexité sociale actuelle rend les relations entre les deux niveaux acteurs/agents plus fluides et plus diffuses... ce qui n'empêche nullement les acteurs collectifs d'êtres présents et efficaces sur la scène publique». Relevant que "l'acteur peut apparaître comme une figure intervenant de façon récurrente sur la scène publique pour faire valoir un point de vue et trouver des modalités pour l'imposer», qu' "aujourd' hui, d'après les lieux, cet acteur peut voir sa pertinence grandir ou décliner», le sociologue belge énonce finalement ce principe voulant que l'acteur soit «une fiction opérative qui n' existe qu'à travers des agents concrets qui ont contribué à lui donner une existence sociale. Il devient un pôle promoteur de sens dans la sphère publique».

\section{Des connotations géographiques qui restent ambiguës}

On remarquera que cette définition de l'acteur dans sa dialectique à l'agent n'est pas totalement éloignée, pragmatiquement du moins, du type de distinction que proposent les auteurs du Dictionnaire de géographie humaine, (Barret, Charvet, Dupuy et Sivignon, 2000), entre l'acteur agent spatial, terme désignant selon les cas un individu, un groupe de personnes ou une organisation auxquelles on attribue un pouvoir d'agir de façon efficace sur l'espace géographique, et l'agent (singulièrement l'agent économique d'ailleurs), terme désignant selon les cas un 
individu, un groupe de personnes ou une organisation dont le comportement économique (ou social) est identifiable, notamment par des données comptables. La question est alors de savoir pourquoi en rester là? Les ménages, les entreprises, les administrations, pourquoi pas les villes elles-mêmes, sont couramment considérés comme des agents (économiques). Un ménage perçoit un revenu, paie des impôts, épargne et consomme en arbitrant entre épargne et consommation et entre différents postes de consommation (alimentation, santé, loisirs..etc..)

Comme les agents qui ont contribué à lui donner son existence sociale, l'acteur interagit avec d'autres acteurs et les géographes aiment à penser que les transformations de l'espace sont le résultats de ces interactions. L'idée est très générale et peut-être reportée à la VIème thèse de Marx sur Feuerbach: «L'homme n'est pas une abstraction inhérente à l'individu isolé, il est l'ensemble de ses rapports sociaux », «biologiques et sociaux » dirait sans doute Edgar Morin. Les mauvaises relations que les hommes entretiennent avec la terre ne sont que l'expression des mauvaises relations qu'ils entretiennent entre eux. Plus géographiquement, on dira par exemple que la péri-urbanisation française est le résultat pour beaucoup du jeu interactif (et conflictuel) des acteurs suivants: les agriculteurs qui souhaitent continuer à cultiver, étendre leur exploitation ou réaliser leurs biens; les promoteurs immobiliers ou des lotisseurs qui projettent des opérations de logement sur des terrains libres et bon marché; les ménages urbains qui recherchent des maisons individuelles économiques; les municipalités rurales heureuses d'accueillir des habitants (et contribuables) supplémentaires, mais soucieuses de ne pas se laisser déborder par les besoins d'une population nouvelle exigeante et nombreuse....etc...

Finalement la banlieue elle-même se présente comme un acteur dans le jeu urbain, parmi d'autres acteurs, plus ou moins centraux ou périphériques, qui agissent plus ou moins consciemment en tant que tels par leurs représentants ou leurs habitants.

\section{Des significations qui se précisent et s'élargissent : l'acteur en tant qu'agi.}

Mais laissons là pour l'instant la géographie et revenons à la distinction entre agent et acteur, telle que censée évoquer le jeu entre les déterminismes sociaux qui agissent sur l'individu (agent) et sa liberté d'action (acteur). Si cette distinction est très parlante, ne constitue-t-elle pas au même temps un obstacle à une pensée relationnelle plus enrichissante? On pourrait dire, par une formule condensée, que le «protagoniste» de l'action sociale est acteur en tant qu'agi. Ce qui reviendrait à dire que l'acteur a des espaces de liberté, mais que les «contours » de cette liberté (sinon son contenu) sont partiellement déterminés par des contraintes sociales. $\mathrm{Ou}$, pour reprendre Norbert Elias, «au centre du problème auquel on se trouve confronté, il y a le tissu d'interdépendances à l' intérieur duquel l'individu trouve une marge de choix individuel et qui en même temps impose des limites à sa liberté de choix» (Elias, 1985).

Norbert Elias fut justement l'un des premiers sociologues à faire de la «pensée relationnelle» son principal outil de travail: «ce qu'on a coutume de désigner par deux concepts différents, «l'individu» et «la société», ne constitue pas, comme l'emploi actuel de ces termes nous le fait souvent croire, deux objets qui existent 
séparément, ce sont en fait des niveaux différents, mais inséparables de l'univers humain» (Elias, 1981). L'élaboration des concepts d'habitus et d'habitus collectif fut essentielle pour saisir le double mouvement à la base de la construction du monde social, à savoir l'intériorisation du social et l'extériorisation du subjectif.

D'autres auteurs ont consacré une partie de leur œuvre à mieux expliciter cette approche relationnelle, à essayer de construire des programmes de recherche et des outils méthodologiques qui en tiennent compte. Pensons à Antony Giddens et à sa double herméneutique, qui consiste à comprendre le sens que les acteurs donnent à leurs propres actions et à en même temps à comprendre "sociologiquement » ces mêmes actions en faisant appel à des déterminants qui échappent aux acteurs mêmes (rôle, système, structure, etc.) (Giddens, 1984). Pensons aussi à Pierre Bourdieu que l'on accuse volontiers de sombrer dans le déterminisme, mais qui dans ses écrits a toujours eu des positions sûrement désenchantées, mais jamais mécanistes, comme le démontre cette citation d'un ouvrage qui date déjà de quelques années: «La sociologie suppose, par son existence même, le dépassement de l'opposition fictive que subjectivistes et objectiviste font arbitrairement surgir. Si la sociologie comme science objective est possible c'est qu' il existe des relations extérieures, nécessaire indépendantes des volontés individuelles et, si l'on veut, inconscientes (en ce sens qu'elles ne se donnent pas à la simple réflexion) qui ne peuvent être saisies que par le détour de l'observation et de l'expérimentation objectives (...). Mais à la différence de la science de la nature, une anthropologie totale ne peut s' en tenir à une construction des relations objectives par ce que l' expérience des significations fait partie de la signification totale de l'expérience (...).» (Bourdieu et Boltanski, Castel; 1974).

\section{L'ACTEUR EN GÉOGRAPHIE}

A ce moment de la réflexion, on peut restreindre notre problématique et adopter un point de vue plus spécifiquement géographique. Si le problème de l'acteur en sciences sociales s'articule autour de la relation réciproque entre le social et l'individu, il s'inscrit plutôt en géographie dans la discussion du rapport entre l'homme et l'espace, entre l'homme et le territoire. Comme dans les autres sciences sociales, ce rapport est caractérisé par un mouvement de «double construction »: l'homme est façonné par son milieu au même temps qu'il le façonne, soit par des interventions «physiques », soit par les représentations qu'il construit du territoire qu'il habite, qu'il parcourt, qu'il vit et qu'il perçoit.

\section{Acteurs géographiques et espace social}

Les principaux acteurs géographiques, ou plutôt «de» l'espace géographique, sont: l'individu (ainsi que la famille, ou le ménage, formes sous lesquelles la décision «individuelle» se manifeste souvent); le groupe, plus ou moins informel ou formalisé (clan, classe, association, lobby, etc...), mais aussi l'entreprise, la collectivité locale, l'Etat. Les deux derniers ont, par définition et par fonction, une action sur le territoire dont ils gèrent une maille, un espace délimité, découpé dans un territoire, et ses éventuelles subdivisions. Mais les autres aussi peuvent avoir une action sur l'espace, bien plus efficace encore. Pris ensemble ils constituent ce 
que nous appelons le système des acteurs au sein duquel se tissent des complicités et se dévoilent des antagonismes.

Bien évidemment les acteurs agissent sur l'espace selon leurs moyens et leurs stratégies, leurs intérêts, et donc leurs divergences et leurs contradictions, qui dépendent, en partie du moins, de leurs représentations - y compris de leurs représentations de l'espace même mais aussi des règles et coutumes sociales, des idéologies dans lesquelles ils baignent ou qu'ils subissent. Il s'en suit des inégalités substantielles dans leurs effets sur l'espace, et des décalages par rapport aux ambitions (et aspirations) réelles des acteurs, ou aux qualités des lieux et des territoires. Ces écarts font à la fois la différence et le mouvement de l'espace, sources de la géographie. Mais il est bien devenu évident à tout géographe que si l'acteur, qui pour lui est toujours réel, et ne correspond pas à une catégorie métaphysique, désincarnée celle des «acteurs désincarnés des doctrines totalisantes », écrirait Roger Brunet, il ne correspond pas plus que l'homme de la VIème thèse de Marx sur Feuerbach à une abstraction inhérente à l'individu isolé. C'est pourquoi le géographe des vingt dernières années s'est beaucoup intéressé aux théories de l'action telles que développées, dans des domaines et avec des concepts parfois différents tant par Talcott Parsons ou AlainTouraine, que par Jurgen Habermas, en mettant l'accent sur les interactions entre individus, sur la socialisation par l'interaction, sur l'autre comme élément d'autodétermination. A ceci près qu'il l'a fait sur un plan spatial avant que de le faire sur le plan social, analysant les actions et interactions dans le domaine spatial même s'il s'agissait de relations sociales.

Ceci dit, il paraît fructueux d' «ouvrir» la problématique de l'acteur géographique en s'interrogeant d'un côté sur la relation entre l'espace social et l'espace géographique d'une part, et d'autre part sur le rôle de l'espace, voire de la ville, dans cette dynamique. L'une des grandes questions du géographe est bien évidemment en effet de savoir dans quelle mesure l'espace lui-même devient acteur, toute la question étant de savoir comment montrer, tout en étant libérés du déterminisme naïf darwinien ou néo-darwinien des ancêtres de la géographie moderne, la spécificité et l'autonomie de l'espace en considérant les conditions auxquelles l'espace construit par exemple «agit» les pratiques qui le parcourent. Ce qui suppose, dirait Sylvia Ostrowetsky (1992), «une redéfinition des lieux de l'articulation du social et du spatial». En marchant sur des œufs, comme on doit le faire quand, par exemple on évoque le langage de la ville en confondant différents niveaux. Faut-il privilégier la matérialité et la texture de la structure urbaine, évoquer d'éventuels urbèmes à la manière d'Abraham Moles, ou s'en tenir plutôt aux discours que l'observateur, l'utilisateur, l'habitant, l'enquêteur, tiennent ou recueillent sur la ville?

Le premier point, qui paraîtra anodin à certains, est pourtant important à souligner: une réflexion géographique digne de ce nom ne peut pas faire l'impasse sur les logiques éminemment sociales, qui traversent l'espace. Si on se soustrait à cette démarche, on risque de réifier l'espace, de prendre les lieux comme allant de soi, existant en soi, de manière paradoxalement abstraite, indépendamment du système de relations («le mode de production en dernière instance» disaient les géographes marxistes), dans lequel la chose étudiée est produite et utilisée. De là l'intention nouvelle de la géographie d'aller au-delà du fait spatial pour découvrir le processus social, de ne considérer les lieux qu'en relation, comme amalgame de faits et de valeurs, de creuser sous le fait spatial pour découvrir le groupe social 
qui l'a produit et reproduit, à la découverte de la racine des comportements dans le monde tel qu'il est naïvement connu et vécu: «retour à l'homme oublié des sciences sociales, à cet acteur dans le monde social dont les actes et les sentiments se tiennent au fond de tout le système » (Schütz). Retour, donc, à l'homme qui dote le monde de sens, qu'il soit tout à tout ou simultanément et pour des gens ou des groupes différents, économique, social, politique, personnel ou symbolique. Le sens (meaning) qui parle de l'existence, celle d'un sujet à la rencontre d'un objet. Cherchant de fait le lien entre la pensée et l'action. En évitant autant que faire se peut d'avoir à s'appliquer le cri des Beatles:

\author{
«He's a real nowhere man \\ sitting in his nowhere land \\ making all his nowhere plans \\ for nobody...»
}

Ce qui est vrai de l'«espace», des «ressources» ou des «comportements» l'est aussi, à un niveau plus général, de l' «objet» et du «sujet» que l'on ne peut plus regarder comme des entités indépendantes, mais comme une relation entre l'un et l'autre. Le sujet structure l'objet alors même qu'il est structuré par lui. En transformant la nature, l'homme se transforme lui-même. L'acte qui consiste à observer est un acte d'évaluation. Les séparer reviendrait à introduire de force au sein de la pratique humaine une distinction qui n'existe pas dans la réalité. Les idées elles-mêmes sont des relations sociales à travers lesquelles la société peut être structurée et restructurée, de même que les concepts et les catégories historiquement déterminés et qui, pour se présenter comme internes à une connaissance qui nous est transmise, sont aussi le reflet du monde au sein duquel cette connaissance est produite.

\title{
Acteurs géographiques et espaces du discours
}

De là d'ailleurs le mouvement actuel des géographes en direction d'une interrogation sérieuse sur la nature de ses objets, qu'ils ont fini par découvrir en tant qu'objets de discours, générant leurs propres ontologies. Tout en nous mettant en rapport cognitif avec autre chose qu'eux mêmes. Eternel débat entre réalistes et nominalistes. "Il est vrai qu'il y a quelque chose en soi; il est vrai que n'a de signification que ce que nous produisons. Chacune de ces positions exclut, à sa façon, leur composibilité même, alors qu'elle est un fait qui découle de la nature du symbolique même» écrit Marie Jeanne Borel (Borel, 1991). Les géographes ont entendu, et peut-être précédé Bruno Latour (1997) et son «Nous n'avons jamais été modernes» et pensé que comme les objets de la physique elle-même, leur objet spatial est bien «un hybride pétri de notre subjectivité». De là sans doute leur intérêt croissant pour les théories constructivistes et les pratiques de l'ethnométhodologie, et, singulièrement, à l'image de la socio-linguiste Lorenza Mondada (2000), leur volonté de questionner, après la scientificité de leur discipline ou ses dimensions idéologiques, le rapport des énoncés à leur référent de manière à mettre en lumière la distinction entre objets naturels, objets produits, objets de discours, montrant «la dimension discursive de la fabrication des connaissances », mieux, la fabrication discursive de la «réalité », leur volonté de travailler sur les configurations de la réalité qui résident dans la diversité et dans l'interaction des descriptions, savantes ou populaires, qui en rendent compte. 
C'est très précisément ce que l'un d'entre nous a essayé de faire, avec Marina Marengo (Marengo et Racine, 1998, 1999) dans leurs études sur les lieux urbains de l'interculturalité et la recherche de ces «lieux de l'entre-deux»plus ou moins favorables à la rencontre entre cultures. On découvre en effet que le rapport à l'altérité a bien une ou plusieurs dimensions spatiales récurrentes, tant au plan descriptif, qu'explicatif ou normatif d'ailleurs, mais sans que jamais on ait pu établir la moindre composante déterministe. Une fois identifiés les lieux qui allaient faire l'objet de leur étude, il fallait passer de l'analyse contextuelle initiale - qui avait permis de légitimer le choix des terrains- à l'observation des lieux eux-mêmes et au travail au sein de chacun des lieux choisis. Mais en se demandant comment décrire et caractériser les lieux qui allaient être nos terrains d'enquête, ils ont considéré que leur catégorisation comme lieux ethniques ou comme lieux d'interculturalité n'était pas donnée a priori, mais qu'elle était produite par les acteurs eux-mêmes dans leurs pratiques. Au lieu de projeter sur le terrain une définition a priori ou théorique de l'ethnicité, il s'agissait d'interroger la façon dont les acteurs concevaient l' «ethnicité» des rencontres, des lieux, des personnes, la façon dont ils traitaient cette propriété comme pertinente, en la faisant intervenir pratiquement dans certains contextes pour rendre intelligible une situation, un événement, un problème. C'est dire que déterminer comment certaines instances, institutionnelles, réglementaires, légales, élaborent et font fonctionner la pertinence des catégories ethniques et interculturelles devait passer par l'analyse de leur discours, de la façon dont ceux-ci produisent une cohérence, une intelligibilité, voire un contrôle de la réalité sociale, de la façon dont historiquement ces dispositifs d'inscription catégorielle s'étaient sédimentés et ont été codifiés, de la façon plus ou moins efficace dont ils s'exerçaient sur une société et essaimaient entre elles. C'est ainsi qu'à l'aide d'animateurs sociaux et en général de témoins privilégiés, il s'agissait d'établir comment les acteurs sociaux ont construit et catégorisé par le passé, comment ils construisaient et catégorisaient aujourd'hui ces mêmes lieux. Mieux encore, et plus généralement, comment ils arrivaient à catégoriser leurs relations à l'espace et à la société dans lesquels ils vivaient et agissaient. Les descriptions données de lieux ne relevaient donc pas d'un savoir désincarné des chercheurs mais d'une activité descriptive des acteurs. En effet, les pratiques sociales des acteurs sont indissociables de pratiques de description, d'interprétation, d'élaboration symbolique qui les organisent, leur donnent sens, en même temps qu'elles en rendaient compte. Les procédures de catégorisation font partie de ces pratiques et permettent aux acteurs non seulement d'identifier des personnes connues ou inconnues, mais encore d'interpréter les activités, les positions, les opinions, les fréquentations comme étant liées aux catégories. Il s'agissait donc de se demander comment les acteurs eux-mêmes sélectionnaient et faisaient fonctionner une catégorie qu'ils jugeaient pertinente pour décrire d'autres acteurs ou des lieux de fréquentation.

Ceci avait pour conséquence que les références à la culture d'origine et à la culture d'accueil étaient négociées, construites de façon spécifique dans chaque situation (à l'usine, à l'école, au bistrot, à la maison, dans les associations, dans les espaces publics..) et ne sont donc pas données d'emblée ni de façon figée. Ainsi, la référence à l'identité culturelle ou à la culture d'origine ne pouvait fonctionner comme une «essence» de l'individu ou du lieu, une «nature; qui les détermine une fois pour toutes et de façon univoque: les appartenances hybrides, les 
syncrétismes culturels, la capacité d'innovation dont témoignent les expériences de migration et d'interethnicité urbaine mettent en crise le modèle monolithique par lesquelles un acteur mobilise la référence à des catégories ethniques, les justifie en les faisant fonctionner au sein d'une démarche interprétative cohérente dans son univers social et culturel.

Ce travail d'observation participante a permis en particulier d'observer comment, dans le détail des interactions, s'élaborent pratiquement des processus d'intégration, d'assimilation, ou de ségrégation qui sont, là encore à définir non pas en rapport avec des modèles académiques mais en fonction de la façon dont les étrangers eux-mêmes gèrent leurs rapports à la société d'accueil, leur inscription dans des réseaux, leurs modes de sociabilité, non pas en général, mais dans la pratique de leurs activités quotidiennes.

C'est dire comme l'observation participante a permis de faire une analyse qui intègre le vécu et les représentations des acteurs, et a également permis de raisonner à plusieurs échelles: celle de l'espace de la ville, de l'espace relationnel des associations, mais aussi de l'espace intime des acteurs qui parcourent, créent, donnent du sens à ces espaces multiples. Je reste néanmoins convaincu que l'ethnométhodologie, pour être pleinement significative, doit être accompagnée par des réflexions sur la structure objective des rapports entre acteurs (individuels, collectifs, institutionnels), déterminée par la position respective de ces acteurs dans l'espace social, s'il est vrai que les perceptions individuelles et les catégories d'interprétation que les acteurs appliquent à la réalité sont partiellement issues de cette même structure objective.

Et c'est bien en gardant à l'esprit ces nouvelles problématiques géographiques que l'on peut essayer de jeter une nouvelle lumière sur la relation entre l'espace social et l'espace géographique d'une part, et d'autre part sur le rôle de la ville dans cette dynamique. Comment faut-il interpréter, par exemple, les mécanismes de ségrégation et stigmatisation de certains quartiers urbains? Faut-il ramener ces processus aux caractéristiques socio-géographiques de ces endroits, à l'action des acteurs qui habitent ces lieux, ou bien aux décisions des acteurs de la politique de la ville? Finalement, quel est le rôle de la ville elle-même? Encore une fois, peuton la considérer un véritable «acteur»?

\section{VILLES ET POLITIQUES URBAINES : LA VILLE COMME ACTEUR?}

S'il est vrai qu'il ne peut pas y avoir d'analyse spécifiquement géographique sans référence au «tout social» (Mauss), alors l'espace ne prend sa pleine signification qu'à travers les rapports sociaux qui y sont vécus et qu'il influence «tant au niveau des effets de conscience qu'au niveau des effets de pouvoir, c'est-à-dire tant en ce qui concerne la construction des représentations de la vie sociale qu'en ce qui contribue à déterminer la capacité différentielle d'intervention des acteurs sociaux» (Remy et Leclercq,1998). Il est fondamental de garder ceci à l'esprit pour éviter de sombrer dans la pensée substantialiste des lieux, qui attribue aux seules caractéristiques (supposées immanentes) des lieux les logiques sociales qui s'y déploient, en oubliant de prendre en compte la configuration des relations qui se nouent entre les différents acteurs dans un cadre institutionnel et spatial donné. 
Comme l'affirme Pierre Bourdieu dans son remarquable article «Effets de lieu», «on ne peut rompre avec les fausses évidences, et avec les erreurs inscrites dans la pensée substantialiste des lieux, qu'à condition de procéder à une analyse des rapports entre l' espace social et les structures de l' espace physique» (Bourdieu, 1993). Et ces rapports sont des rapports de correspondance, s'il est vrai que les deux espaces se caractérisent par un système de localisations hiérarchisées (en fonction de la distance physique et sociale). «La structure de l'espace social se manifeste ainsi, dans les contextes les plus divers, sous la forme d'oppositions spatiales, l'espace habité (ou approprié) fonctionnant comme une sorte de symbolisation spontanée de l'espace social. Il n'y a pas d'espace, dans une société hiérarchisée, qui ne soit pas hiérarchisé et qui n'exprime les hiérarchies et les distances sociales, sous une forme (plus ou moins) déformée et surtout masquée par l'effet de naturalisation qu'entraîne l' inscription durable des réalités sociales dans le monde naturel: des différences produites par la logique historique peuvent ainsi sembler surgies de la nature des choses (il suffit de penser à l'idée de «frontière naturelle»)». (Bourdieu, 1993). L'espace social, donc, se retraduit toujours (mais pas par un lien de causalité automatique) dans l'espace physique. La position sociale d'un acteur s'exprime toujours aussi par sa localisation spatiale, l'exemple extrême étant celui du SDF dont l'absence de domicile retraduit son «inexistence» sociale.

Ceci étant, la question de l'exclusion urbaine peut être problématisée par rapport au thème de l'acteur dans les sciences sociales, et plus particulièrement de l'acteur en géographie. Il ne s'agit pas, dès lors, d'aborder le problème de la ségrégation par les seuls outils de l'analyse spatiale, en mesurant et en cartographiant le degré d'homogénéité d'un espace par rapport à une certaine catégorie de personnes. Il s'agit plutôt de montrer que l'appartenance à des espaces concrets et l'organisation de ceux-ci jouent comme élément constitutif du pouvoir des acteurs sociaux et, dès lors, pèsent dans la transaction sociale. Les acteurs des quartiers stigmatisés, par exemple, sont doublement dépossédés : leur statut socioéconomique les prive d'une part des instruments nécessaires pour participer aux différents jeux sociaux, et d'autre part ils sont d'autant plus «exclus » que le fait d'habiter un quartier stigmatisé les dégrade symboliquement (Bourdieu, 1993).

Ces quelques éléments d'interprétation nous conduisent à reconsidérer le problème des acteurs dans le cadre de la politique de la ville. Comme nous en convaincs Lorenza Mondada (2000) dans son beau Décrire la ville, «la ville est bien une entité matérielle et immatérielle configurée par les pratiques des acteurs qui l'habitent et la fréquentent, y travaillent et y vivent, ainsi que par leur discours, qui la qualifient et la requalifient, lui confèrent un ordre, s'y identifient ou s'en écartent». On entend parler de plus en plus souvent d'«acteurs compétents», terme qui est majoritairement utilisé dans les approches participatives de la politique de la ville pour déterminer qui est habilité à participer aux processus de décision. L'idée qui sous-tend cette expression est que la «compétence» est en lien étroit avec la connaissance du terrain. Ce postulat est sûrement vrai en partie, et a permis de donner plus de voix et pouvoir aux associations travaillant en étroit contact avec les populations marginalisées. Pourtant, il serait illusoire de croire que les acteurs qui vivent dans des situations d'exclusion, et qui ont donc plus d'expérience de terrain que quiconque, sont automatiquement considérés comme «compétents» par les autorités publiques. Au contraire, par le processus de 
double dépossession que l'on a décrit plus haut, les «exclus » reproduisent, après l'avoir intériorisée, l'identité dégradée (dominée) qui leur est continuellement renvoyée par leurs appartenances spatiales et sociales, et sont donc souvent bien loin de réunir les atouts nécessaires pour participer à la politique de la ville. Leurs «compétences » sont rarement valorisées du point de vue institutionnel, car elles ne correspondent pas aux «compétences» dominantes (prestige, capacité de négociation, maîtrise du langage bureaucratique, etc.). Par conséquent, les autorités ne peuvent espérer entamer un véritable processus d'intégration politique des habitants des quartiers défavorisés qu'en remettant en cause leurs propres critères de compétence, ce qui est ardu mais pas impossible.

Le problème de la «représentation politique» des classes défavorisées nous aide aussi à comprendre la question, plus large, des « violences urbaines ». Maints auteurs, parmi lesquels citons Michel Wieviorka et François Dubet, ont expliqué l'augmentation des violences notamment par l'échec de la représentativité des institutions républicaines, qui seraient désormais incapables de remplir leurs fonctions classiques (assurer l'égalité individuelle et la solidarité collective). Dans ce cadre de déstructuration politique et de désocialisation des individus, il faut, selon Michel Wieviorka, analyser la violence par une approche compréhensive qui intègre les acteurs de la violence, leurs attentes, leurs désirs, leurs frustrations. La violence urbaine, vue sous cet angle, ne serait donc pas seulement un acte gratuit, mais un acte traduisant une conflictualité qui ne trouve ni d'autres canaux pour s'exprimer, ni de modalités de traitement politique des aspirations qu'elle met en forme. «La violence, ici, indique le désir de modifier une situation devenue intolérable. Sous certaines conditions, elle peut éventuellement constituer l'annonce d'autres conduites, non violentes (...). Ainsi, il arrive qu'après une émeute certains (...) s'engagent dans une activité associative, (...) deviennent des acteurs politiques de la scène locale (...). Un tel cheminement impose de voir dans la violence un moment, éventuellement fondateur, qui n' enferme pas les acteurs concernés dans la seule logique de crise, et pas davantage dans celle du calcul et de la logique instrumentale.» (Wieviorka, 1999: 19). Ainsi, les émeutes seraient à la fois post-politiques, car elles témoigneraient de l'épuisement des modalités de traitement politique et institutionnel des demandes sociales, et pré-politiques, en tant qu'expression d'un manque politique en matière de traitement démocratique de demandes sociales. À la fois mouvements expressifs et instrumentaux, elles pourraient donc permettre aux exclus de réactiver un processus politique non-violent, dans lequel seraient traitées les demandes civiques et sociales qu'elle est venue signifier.

Cette interprétation est particulièrement séduisante parce qu'elle évite, entre autres, de donner à la violence une portée normative (vue souvent comme le moteur du changement historique, comme dans le marxisme), ainsi qu'une vision apologétique. En même temps, elle nous paraît insuffisante, en ce sens qu'elle évacue tout le problème des rapports de forces qui conditionnent très largement les comportements des acteurs et structurent le monde social. En voyant dans la violence contemporaine des conduites qui pourront élargir l'espace de la participation politique et marquer la liquidation des rapports sociaux actuels, Michel Wieviorka n'alimente-t-il pas l'illusion de la fin de la société des classes et l'avènement d'une société parfaitement «horizontale» (Touraine), structurée non pas par des rapports des forces hiérarchiques, mais par le clivage entre les «ins» (ceux 
qui participent pleinement aux activités productives ainsi qu'à la vie sociale et politique) et les «outs», à savoir les «exclus»? Mais cette vision est dangereuse et simpliste, car (si on veut jouer avec les mots), il y a, parmi les «ins», des «ins» plus «ins» que les autres, tout comme parmi les exclus il y a ceux qui le sont plus que d'autres. En effaçant la complexité hiérarchique qui structure les relations entre différents acteurs sociaux, elle contribue à méconnaître la domination qui s'exerce sur les couches plus défavorisées de la société.

Pour conclure sur ces problèmes de «représentation politique urbaine », nous aimerions reprendre une interrogation formulée plus haut, et latente tout au long de cette réflexion/discussion: et la ville dans tout cela? Peut-on vraiment considérer qu'elle fait fonction d'un véritable «acteur collectif»? Nous ne sommes pas convaincus qu'on puisse véritablement considérer la ville comme un acteur collectif, si l'on entend par acteur collectif un groupe qui s'est constitué dans le temps pour la promotion et la défense d'intérêts communs. En revanche nous pensons pouvoir affirmer que la ville, en tant qu'espace où se cristallisent des contraintes sociales et spatiales mais aussi en tant que lieu privilégie d'échange et de liberté, est l'endroit idéal pour la constitution d'autres acteurs collectifs. En effet, ceux-ci construisent leur identité commune par et parfois contre la ville, dans ce sens qu'ils doivent se confronter avec un système de relations foisonnantes plus «dense» qu'ailleurs, ce qui comporte une multiplication des contraintes mais aussi des interstices où construire les possibilités d'une plus grande liberté. Liberté qui, pour se réaliser, doit pouvoir donner «la maîtrise réelle des mécanismes qui fondent la méconnaissance collective; en sorte que le travail d'objectivation, nécessairement collectif, qui rend explicite ces mécanismes, loin de constituer le chercheur en une sorte de juge suprême, supérieur et extérieur au champ qu'il analyse, prétend seulement à restituer aux individus et aux groupes le moyen de se réapproprier cette vérité que l'on dit objective parce qu'ils n'en sont pas pleinement les sujets et qu' elle ne peut être produite que par un travail collectif. S'il n' est pas inconcevable que les agents sociaux s'affirment enfin comme «maîtres et possesseurs de la nature» sociale, c'est-à-dire de leur propre nature, c'est qu' il n' est pas impossible d' imaginer des univers sociaux où seraient réunies les conditions pour que la connaissance de la vérité objective, cessant d'être l'arme par excellence d' une lutte pour la domination, devienne le principe d'une maîtrise du groupe par lui-même.» (Bourdieu, 1998).

\section{CONCLUSIONS ET PERSPECTIVES TRÈS PROVISOIRES}

Quant à conclure plus généralement, et sur l'ensemble, disons simplement ici qu'il s'agissait pour nous d'entrer dans le débat et de nous mettre en situation de partage face à nos collègues des autres sciences humaines. Notre bref inventaire nous a sans doute pas permis de franchir une nouvelle étape dans l'analyse réflexive de nos propres disciplines. Il fut néanmoins pour nous l'occasion de renouer avec des problèmes «fondamentaux» des sciences sociales qu'une attitude bien trop arrogante pourrait considérer comme résolus une fois pour toutes.

Reconnaissons que le parcours des géographes a été caractérisé par plusieurs «virages» méthodologiques, des ré-orientations continuelles, à la recherche de 
changements paradigmatiques dictés par l'évolution de la connaissance et de la science, à la recherche continuelle résultats qui fassent sens, bien sûr, mais aussi de nouveaux parcours épistémologiques. Dans ce contexte, la réflexion sur le rôle de l'acteur en géographie, - et il en va sans doute de même en économie, et dans les sciences sociales en général -, constitue une occasion privilégiée pour entamer ce travail réflexif dont on a parlé dans l'introduction. Au risque parfois de se tromper, ou de choisir des outils dont on ne saisit pas encore complètement la valeur ou les dangers heuristiques. C'est le cas, par exemple, des outils ethnométhodologiques, que Marina Marengo et Jean-Bernard Racine ont largement utilisés dans leur étude sur Les lieux de l'interculturalité, réalisée dans le cadre du projet PNR 39 du FNRS, Migrations et relations interculturelles. Sans vouloir y revenir ici longuement, notons simplement que les deux auteurs soussignés ont néanmoins, et malgré l'intérêt des résultats obtenus, longuement débattu des limites, voire des dérives possibles d'un travail d'observation participante, certes passionnant et riche de différents points de vue.

Il s'agissait, entre autres, d'établir comment les acteurs sociaux ont construit et catégorisé par le passé, comment ils construisaient et catégorisaient aujourd'hui ces lieux «hybrides» ou d'entre-deux, d'autres parlent d'interstices, que nous avions provisoirement définis comme lieux potentiels d'interculturalité. Comment les acteurs sociaux, étrangers ou autochtones concernés, arrivaient à catégoriser leurs relations à l'espace et à la société dans lesquels ils vivaient et agissaient. Nous ne voulions plus que les descriptions données de lieux relèvent d'un savoir désincarné des chercheurs mais d'une activité descriptive des acteurs. En effet, nous rappelait volontiers Lorenza Mondada (2000), - et nous résumons ici sa pensée- les pratiques sociales des acteurs sont indissociables de pratiques de description, d'interprétation, d'élaboration symbolique qui les organisent, leur donnent sens, en même temps qu'elles en rendent compte. Les procédures de catégorisation font partie de ces pratiques et permettent aux acteurs non seulement d'identifier des personnes connues ou inconnues, mais encore d'interpréter les activités, les positions, les opinions, les fréquentations comme étant liées aux catégories. Il s'agissait donc de se demander comment les acteurs eux-mêmes sélectionnaient et faisaient fonctionner une catégorie qu'ils jugeaient pertinente pour décrire d'autres acteurs ou des lieux de fréquentation.

Ce travail d'observation participante nous a en effet permis d'observer comment, dans le détail des interactions, s'élaborent pratiquement des processus d'intégration, d'assimilation, ou de ségrégation qui sont, là encore, à définir non pas en rapport avec des modèles académiques mais en fonction de la façon dont les étrangers eux-mêmes gèrent leurs rapports à la société d'accueil, leur inscription dans des réseaux, leurs modes de sociabilité, non pas en général, mais dans la pratique de leurs activités quotidiennes. L'occasion, bienvenue, de faire une analyse qui intègre le vécu et les représentations des acteurs, et qui nous a donc permis de raisonner à plusieurs échelles: celle de l'espace de la ville, de l'espace relationnel des associations, mais aussi de l'espace intime des acteurs qui parcourent, créent, donnent du sens à ces espaces multiples. Il reste qu'au final, nous demeurons convaincus, au vu des résultats obtenus, que pour être pleinement significative et utile à l'action, l'ethnométhodologie, telle que du moins nous l'avons pratiquée, doit être accompagnée par des réflexions sur la structure objective des rapports entre acteurs (individuels, collectifs, institutionnels), déterminée par la position 
respective de ces acteurs dans l'espace social, s'il est vrai que les perceptions individuelles et les catégories d'interprétation que les acteurs appliquent à la réalité sont partiellement issues de cette même structure objective.

Institut de géographie

Université de Lausanne

\section{BIBLIOGRAPHIE}

Akoun, A., Ansart, P. (1999), Dictionnaire de sociologie, Paris, Le Robert, Seuil.

Barret, Ch., Charvet, J.P., Dupuy, G.,et Sivignon, M. (2000) Dictionnaire de la géographie humaine, Paris, Liris.

BOREL, M.-J. (1991), «Objet de discours et représentation», Langages, no. 103, p. 36-50.

Bourdieu, P. (Dir.) (1993), «Effets de lieu», in La misère du monde, Paris, Seuil.

BOURDIEU, P. (Septembre 1998) «Sur l'objectivation participante. Réponses à quelques objections », Actes de la recherche en sciences sociales.

Bourdieu, P., Chamboredon, J.-C., PAsseron, J.-C. (1968) Le métier de sociologue, Paris, Mouton / Bordas.

Bourdieu, P., Boltanski, L., CAstel, R. [et al.] (1974) Un Art Moyen : essai sur les usages sociaux de la photographie, Paris, Ed. de Minuit.

Elias, N. (1981), Qu' est-ce que la sociologie?, Paris, Pandora.

Elias N. (1997) Logiques de l' exclusion, Paris, Fayard.

Elias, N. (1985), La société de cour, Paris, Flammarion, Coll. «Champs».

GidDEns, A. (1984), The Constitution of Society: Outline of the Theory of Structuration, Cambridge, Polity Press .

LAtour, B. (1997) Nous n'avons jamais été moderne: essai d'anthropologie symétrique, Paris, La Découverte .

LeFeBVRe (1972) La pensée marxiste et la ville, Paris, Casterman.

LOSHAK, Danièle (1986) «La société civile: du concept au gadget», in COLLECTIF, La société civile, Paris, P.U.F.

MondadA, L. (2000) Décrire la ville, Paris, Anthropos, coll. Ville.

OSTROWETSKY, S. (1992), Le sens spatial comme acte de langage à travers le faire et le dire urbain, in Pellegrino, P. (éd.) Culture architecturale, culture urbaine, Paris, Economica.

PInTo, L. (1998) Pierre Bourdieu et la théorie du monde social, Paris, Albin Michel, coll. «Idées».

MARInGo, M., Racine, J.B. (1998) Migrations et relations d'interculturelles: Les lieux de l'interculturalité, Géographie et Cultures, no.25,, p. 22-37 (en coll. avec Marina Marengo).

RACINE, J.B. (1999) The foreigner and the city; from co-presence to Interaction, in Search of intercultural Places in Lausanne (Switzerland) (avec Christophe Mager), in A. Aguilar and I. Escamilla (eds.) Problems of Megacities: Social inequalities, Environmental risk and Urban governance, Universitad Nacional Autonoma de México, UNAM, Commission of Urban Development and Urban Life, IGU. pp. 477-498.

RACINE, J.B. (1999) Rapport à l'espace, rapport social et rapport à l'autre dans la ville cosmopolite: questions à la géographie urbaine, in Collage, Périodique d'urbanisme et d'aménagement du territoire, no.4, pp.18-21.

REMY, J. (1989), «Comment problématiser le changement social?» in Le mouvement et la forme, essais sur le changement social, Hommage à Maurice Chaumont, Bruxelles, Hommage à M. Chaumont, Ed. Facultés Saint Louis.

RÉMY, J. et LECLERCQ, E. (1998) Sociologie urbaine et rurale. L'espace et l'agir, l'Harmattan, Paris. WiEviorka, M (1999), Violence en France, Paris, Seuil. 